\title{
ASPECTOS LEGALES DEL TERRORISMO
}

\section{Luis Bramont Arias}

Abogado, Doctor en Derecho. ex Vocal de Ia Corte Suprema de Justicia y profesor de Dere. cho Penal en la tniversidad de Lima.
suMARO, - 12 Lmitociso del toma. 2.) E teeroriamo y ou problemática. 3*1 Textos lecolien as Mortologa del dello de tenconemo. $5^{2}$ y Core: derociones solane ef digrifieado do la lutela juridico of lomodemo gomónco. 7 Tertarismo agiawodo in? Concerto poradeingul. (P) incitu-

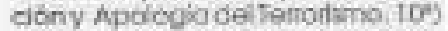

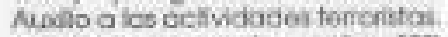
11\%) Exmentes o deruanies. 127 Aspecto Procesor.

\section{F⿻) HAMEACCION DEL TEMA}

Elaspocto logaldei ferio rismoes uno de las rocinde can que se

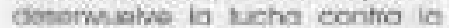
Mokirkia y of testor, que en los illimos anos has crockio al extrerio de cairrir in rol prolagónco y commerlinesen ot contro do lapreo. cucación ciusodare.
B pioblentio del sevorisio es mul corrpleja D Goblemo ie defienco aiskamente pole ig sut

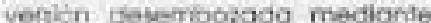
und parciol respuesilo poiveul $y$ minat. pero hosta la fecha no tre doimogdo und estratego global paro ertientatio. puat on nuestro sociectaderisterung derional clate buctoti del poder de deside aboie la disignación de los recurtos heceacuices pora ia segaridad cutheal. reidisc y económica de los crupos sociales y de los ciudadanoe

A protiona to vincuion mot Ruse cuestionce de oeden peltica.

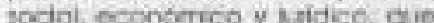
no eu-posiblo csendidera on este astudio per ationa nos krilamos o excrmina las do indiole pafdice pond wero docit, las referentos $\mathrm{c}$ coltio de temoritumo. 


\section{2) EL TEERORISMO Y SU PROALEMATICA}

Lo videncia que vito el pors, per we escaia y profundidod. atento seriamente contio nuserro sistemo peltice, porionco on nisego io $\alpha$ gonzoción dol Estado cues se insclro y base an las pinctaios de und Republeaeccial y derncenctieg de Derecho (cat. 79 da la Constrit cbri)

B lencilstio os un fencriveno que to viono gostandolentamerve y qua. kualmente, esta lamats o dxmintil lexternente, el se aploan sernedios dernto do una estratege clobo:

tos cenductas vidercos deben sor anclibodas en el contexto go. noncl de los problemes socicties o que hocer frente las socicidnclos eontecriocíanect. Detorminadas fractores corro et decarnvelso is manginacion socicl, is frustrockr humana o la coriducta pollica. ondta otics stuociones. Giecn $y$ pipvocan una verdedela khoestruchira de la violencio.

En is kuctio contro otton con ductos violenilas se ha de clerecar far. descle lixigo hat actitudes pit ramorito emociancias. Cerecen de eflacorio bipeno demuerte oe end areciriento de las penos phivothas de whertod BECCAPIA enemb ciue no eat la movor crueddort de kns peras, sino su incriblidad bo que constinume un oma elicas en b fucha contro ia eriminglinaci. eato es, gue ba pesion te cumplan de verdad La certeea de un cati. po. Cunque este sea mociarnia

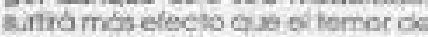
ofrr man temble urvida a ic espes. urus de la mpunidad o. de ay in cumplimbnto. tos turciones pre ventivos de ka pena no dependen tanto de io ievericiad de feria cuanto de is efrocz persecracich pelicios ds delos. Nopidez en suen hiciamieriloycorlosa onel tarpl. miento de la canclona mpluesta.

En ung Peptolices sockat y de-

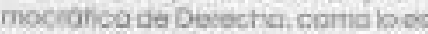
novelferi, et Eerachs panalidebe

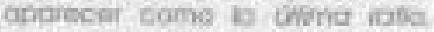

dobo hala'se siempre en uifmo lugar $\gamma$ entrar en juego fan sdo cuardo resulia inctspensabio pasa el manterimianto del oncten juridit. coy dela poz ciudodona. Envithad del principio des interverscikn mont ma dado la diusion de las pericas. cue afecton g los bisonin mos ple. ciosise de la persono, el Devesho ponal dobe interverif solomente cuxricto residter inouficientes otros medica mence dásticos.

La lusho contic ef ferraismo exige la actuación en cuatro trve ins ciforenton: a) mayor astuidad social en la prevencien; bi mays colchoracion leojsatur e) maycs efisociapolcial. y d) maner mode. rocion y rapidez en las ceoisiorves juciciales.

En suma conejderado do esta menewa ef problomo del terrevime

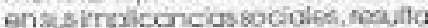
Pued protonder tescivarib sabo of

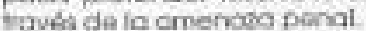

\section{9) TEXTOS IEGALES}

Los textos básicos de 10 logialo. cion penjann rekgtho oi deato cie torroimm tom

a) Lo Ler N 24063, de 7 de at

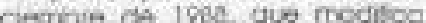

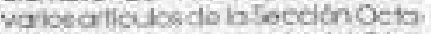
ma' 'A' dellesorsegundo dei Cdai. copericl.

b) La Ley N 2455l, de 19 de morzo de 1982 cince ath 2sia. 28su, 286C. ¿2880, 2nee (nco) y 2bof, handido osegandos porlatey Ne 24063

o) La Ley $\$$. 24700 de 9 de juna ce $10 k 7$ mbre noming cleprocodi rrdenta mon io imestigación pol cia la ir truccion y el uegarrichto dadotlos oxmetidoe oonprepobato lenoristo

d) Lot orn: 2, inc 20 y 169 da ia eonstikgion. aue cortetbyon io icksy mesupuseto do los kryes on fetionrente cilodas
Las kaves han tomoso eemo fusente of Decreto Legliativo N 40 do iD de rrazo de 1981: is ley $N^{2} 24851$, de 19 de morze cs 1987 . la Ley Orgonico cle CAdigo Penal para Espana do 20 de diciembre de 1 sas, yol fstatuto poraladefen. sacie la Dernocrocia en Colornbig. amedido meficrite Decteto $\mathrm{N}$. IBO, de 27 de enero de 1968.

\section{7) MORFOLOGIA DEL DEUTO DE TERRORISNO}

to Loy N 24953 contiana las \&4 giventes delitos:

- Tervorimo genariso iort 28BA. 28de)

- Ternorismo agravado 〈art - Conclerto para delingur (a)t.2sac)

- ncisacibon (art.2880. primeod partes $x$ Apologlo del temsísano (art, zuéD, seourda pato).

- Aimilo o las octimictades terro istas fel mee ha tido madifioode por b Ley N 2495s.

\section{CONSIDLRACIOHES SOBSE EL} SIGNIFICADO DE LA TUTELA JUbiolCA

Objero ganonico of is hitelo perial. en tolación a bi devitos co. mofidio conpropos to temalata es elvileces derestodo por el montere. tranto de ia liangisickad pribleco contra kxy artas cue, al ptoduci estrages ferrotistas. conciortoe

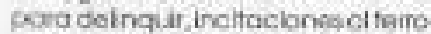
Armo o audilos a kas netivergades teilicristos, sxe tonsentimiantos de preocipsarisin trielpueblo oenun soctor de ex.

La tranguldod ocklico kery que enterisdanip en ias monilastoreknes eclectivian od la vita pocibocilico en coerfuimidad a la orgoninoción irehucional oxtsterte. De gxi que sualecdian torrbien repencuteren in sopurkbat dei futodo y taritikn pueden peducirta comernerscios: 
cen la securidad potblica $y$ darse cosos de concurso idoalo de consuneion. Por torito, los celios do lenertiomo son actes pl.xioforytucs. pued afecton a clierentes tianos juidicos.

\section{TERRORISMO GENIRICO}

a) Descrifocidon fíplea: nenfe iogat

Lo flgurt do este delto so hald concebida on el ot, $283 \mathrm{~A}$ det $\mathrm{CH}$ de la solisinte manera:

"Sand roperritido con poritencia fonemenox de guinco ancsel que provocasa, crecra o mantuviero provocasa, clecra a manliwvera terrer en lopoblación o o unsector do ollo, tectizando par medio de actos contro lo vido, el everpo. if scludi ta ibernod to roguficlod perasenal o la integridad fisica do la peesonas at patrimorio do betae. centrale segurictad de los ecilinelos pitticos vicso medios de comuni

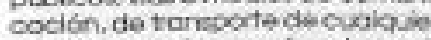

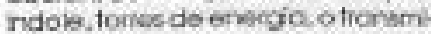
ción. instalaciones motices o cuct

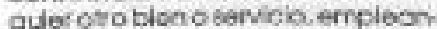
do métodoe viotentos, ammamen tos. matecics o artefoctos emplos: vos o cuakalor ofror mesto capa: de cousar estrogos o do ocasioner gave penvibacbri de la tancat. dad póbioo, de alectaries teicocio nes frlemcicionsios a la eoguridod sociol o eotald

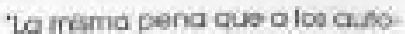
ies moleriales dai devila, se colf con't o los inaligndicres o autores ntelnctucies, cooutcees y combil. ces

'Fi Juez poda dority. ka pona

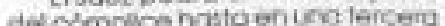
parto de la ropiotibn ck o imparga of outar del hectiv purbab. 9 ig peradis herhopunbio nesolaide hternarienio. la pero di complce. essa la de penitencialia no menor de dóce ufres.

Esta regio reproduce, en cieifa

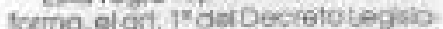

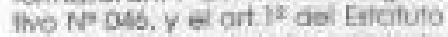
para la Deforiss de la Democrocia do Calombla.

b) Taloidad objetvo

suleto actiro puado sor cual. quier pensena quo proctica lo oscich prohibida por ia nortna. 0 seci el crue ejecuta lo ocolón consh. derada cello do la ley. 5 pertene ciera una orgovizacion pora 00 motor actos do ferrociemo, sotá de cplicacion el he a) det art.28e日.

sulato posivo es la eclectuidad O cl Estado, feriendo en cuenta c bien yides ofervido. como se acobo de sostener.

In canducto que confizura. eocin la ley, ol delito de temcrismo. consiste en 'provocat, crear o mentener ec estodo de zozoblo.

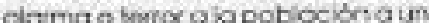
soctorde ello madionheroctos que pongan on polgro lo wida, el cuatpo, la solud. Ia ibeitod, Ia spguriday de kneperenceso el patrimorto de elleso lo seguringe de ke odifcios publiset. vics o modics de comuricoción trarsporte. Pones do enesgio e trosmison. Inefalacio res motnces o cunalquiser otro bien o servicto, valérnasvie de mótodos Wdentos, arnamentos moterins o criofactos omplostuos so cuóquer oho mecla capaz do couser eata oos o de ocorlonar grove pertu. bocibri de lo tronquildad putolico. de alectar las rabciones intemo cioncles o ka secturidad socio so esilator:

to prmerc que impled lo o. ción delictho ea in esistoncio ce

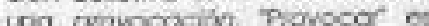
realoner astoe o mocer use dos medios iobreos para couver zatiobra. olome o lernar en la pobiacion. conliene en $=51$ a la "clisociom" y

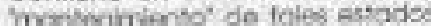
electimos esdecr, "Frovocor sigri. lod hocer nocer val doleminat:

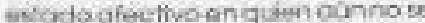

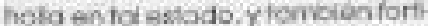
foro in artodo atoctivo ya vovitanfar.

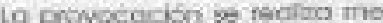

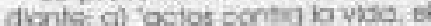

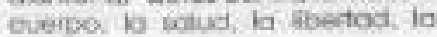

seguridod perwal o contra el po. trincrio do detas", rétendos a los clellos contro la seguidod piotca (5ecclón Seotima dei CP), quescio pupdo ser lasionada por la creo. cín de perkro pano los benes onotodos b) octos centralosegu.

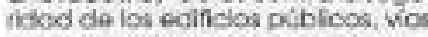
ornedicos de comurcacóno frons. porto de cuolaterindole lams, 268 . 270 de ( CP), forter do orvigio a ncartision, hetclaciories snotricesc cucloued otro blen o servicio (art 271 del CP) Estos actos son copoces o idónocs paro crecr 2obebro, alorma o temor en la po. buación No es nocesuio. en consecuencio, quo do produreca teat mente los rmaneioncicos estodos alectiwce

Segúnibley, el ogenne dol dolto do tortorismo dabe cometer ks octos onlos tericrodgen remplacondo mátodos viclentes omomentos materias o otefactes explosvas cidalas oc ofro medo: o) 0000 ? de counor eethagos: b). casoz de ocosionar grave pearturbocion de is frunquildod puedico of corsor de aloctar lat relocignes nterracionoles: di copoz de ofector lose guridod socal o esigtal Destoca-

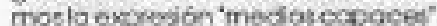
para enfalizar que no es nacesanis que se produmcon estrapos. que te perturbo lo tronquilidad putefeo. OHA $\%$ afecto las molaciones intes. nacicnoleso que se ofectelasegu. sidod socisl a estatd. Fs bastconto qua se compnuebe cue lou medise orrolegoios son kenece para pio dixcir clethan sendtadios.

La provococión dete ser pubteciesto on hocks anle in Mimcro ndeteiminado do hdhtducs o en trimg gie pusoda sar pencirido por estor. $l 0$ que se requere os quo oog difida ar patil. co. pues hay m postlixdad de give of cicmoccroo sed ung vela $16 \mathrm{im}$

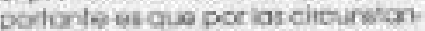
bice en que es fomuado. puede ser cocphodio por in nimano indo-

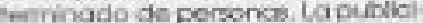
coxd sertos de clarto ndeteerrino. clon en los destinataiks ia lery cent crefomento expreso gue el delto somsidian piovocer wovobia. da maserset en krpoblacion o a un 
secter de eiks:

Lo provococian debe estat onouminada a ptocurar "zowobea dicmna o ferror'. For 'sazabsa' en fiende el Diccioncrio de la ienauo cepcridia. lo inquietud, aficelon y congoja que no deja sosergar, o por of riesjo de amanana o par e mal que yase podsce, 'Alarma' en incliasud, doscsoskge, sobrese? to. Ternor' es miodo, ospanto gran de ante una amenza o pelgio para ciertos sectoras sceleles o pobkictón determincodo o qie pueden desorgarizat una estrus. tuna ecronśrrisa social o polticla.

\section{c) Trablobog subletivo}

B tonotarno toquisere dolo $\mathrm{kec}$ drecto o de consecuencios recesarias), que conesto on la welurked de saiear zazobra. alarna o le. mer medianto los octos previstoe porla loy.

Esta apecto tucjativo de la firi: sodad corstituve la dforoncia con las dalios centro ta sagurldod putblica y estabiece la naturdede muy particlatar del deilo ca temorio mo. 5 nose lograra onla potylaciś ef estodo de zazobra, abirng terrer, el cist to podría ser tiolifeode como contra bsegundad pCCfica. pero no como temcriaria De obo aclo en bos clelitos contro lo tian. quilidad poelice. id "elaming" este coferiba a lo amenciza de la come. dóndeundelito, en tanto que ene dolito do forrorismo, el estodo de nonokera. examna o tertor de if población. es alos irherente a ka comison delictiva pues como gnoto VILAVICENCIO TERRETOS "debe tentarse o coneurrirse in ittrogo Cdemumbre. emplosion. ole) y no edlo amencorar centod. lacaila io simple giriengesa de

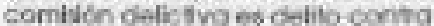
ia tronquisidad pabica (ort $281 \mathrm{CP}$ ) o contig in thenlod individuel corts. 222 y 224 CPF $^{\circ}$ (1)

6) Ginador de descomola provici poción concumay penotiod

Ekte dello so caraung no blen surgo la scwobro, la diamo o es terrer patilco, con íg percepción de la recizackion de las cotos ideneos pera suscitale, por pote de unnürnero detemnado o indeterminado de pessonas.

La Tentafivg es posible.

Respecto o la conarticisackin erminces, is lon ditingis o las oulotes (co-outores y gutores mediatos de los patidipes en el delito de feriofiano Crstiogción. ausiladotos nocesarios y compli. ces). Para elos valen let normcz comunos (at. $100 \mathrm{y} z \mathrm{del}$ CP)

Esto delso puodo concumy con otros focr elomplo, rebo) en curvo coso deben obervare los fegre estoolecidas en et tituls xil deil Li. bio Primero del CP lat. 3 ce lo len $N^{2} 246513$.

in penapora loaculotes y parfl: cipes es peritenciaria do 15 o 20

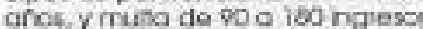
minimas vitolas a inhabiracion abeoluta a interdiceidn civi durarto la condena $y 5$ unos pestediore. a ela (art 268f)

Lepeng datedmcice drube, po reglopenerol. sar idervico o lade autor doldedilo. pero paralos cases do oucopción se toculta of juoz poro derminuit la perno dé córrol ce hosta an in toticio de la q.a im. penga ol outori y asta atonuación es forcosa y se otorga en la fomma de commutacion cuando se trata de hechos a bes cue bi ley coslige coninlemartiemp, encusys coso ka perno serd de perihenclaria ne mencr de 12 oncs citmaparte de an. 268A].

\section{TH TRRORORMO ACRAVADO}

A)

Descriación foica: nuente

Lo ficua der ferroremo garcono 30 it hola conoebioxa en ed art 2685 de sa sipulente moncra

to perve serd:

"c) De pentercionia no meno de dincischo conos s el aosnte perfenecien o uno orpanbocibe. qua, para lorgur the trike cuales. quere ay secon, iftice corrs. mecio el delito do tororiame tipit. codo en ef articulo anterer La pena serddo intemamiento. cuan da el opente pertereciere a la orgarizución on calidna de inte. cobocila o miambea directriz.

b) Do peritenciaría no manor do declocho ones si eerro efocto deidelito ve prodtideronlesianes en personce o derios en blernes puteti cos o pelwodos:

c) De peritenciario no mance deciesicctio âlos.sisa hickereperticlpar a meneres de edod on la cornkbon del cello:

d) De penitencictía no monor do veinte crkis sid el duno on ioe bienns pobloces o primoctes implde. ie lofal o parciamente los senicios esonciales do ia potoloción

'o) De internorrionto, cuando con firses temoristos so cxeconvionert o secuestrate personas porg cotener oxcoxcalaciones de deteridos - cualquír ofro vantaja hdekida porpartedelagutoridod opaticu. bresy cuando, con identca frekdad.se cporkeirone ilcitomonte de vetibulo odreo: ocubtico o terres. to, noclonal o entrarieso, o altero. re su itnencrio: o cuarvdo la extarsurn esecuectio tumiore come fingliod la obtecrebon de dnero ble nes o cualquier otro ventola: $y$

i) De ntemramiento, cuando se causcre miserte o lesiches graves:

Estarogla raproctuce.cos hercalmonts, al at. Ze del Dercieto legls.

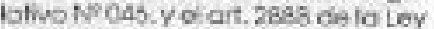
NP2551

El teiraizens basice se orgava: a) poriaexridaddola tor:biecrs: iesutodo. c) por el mock de comt. son; y di por el mecfo y fn dol oufar

Entazon a lo coldod dal autar. conarreglo atine " $a$ ' detart. 2898 ,

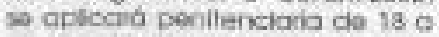

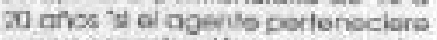
a uno onocibacison gue pora lo. gar this fros, cuglesuiera qus secan, ultico corno medis ex deliso

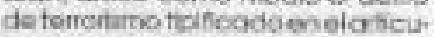
b-anlarice', y be apleada hturra. mistio. Seucurdo of azante perta. reciele a la ergarioodibn en cosi. 
dod de jete, coboclib o miember diectre. If egenta actúa como "beiteneciente' a und orgcrizocibry cuando lo hoce per irenda. to de la omgarixación. pudendo inclusive ser una sola ponofo. es docit. lo que se conoco con e nombre de 'ankdod a una orgarizaclon. Si el perteneckente ourd orgonización getuara na por ingandets o acuotdo cie la orgari. tación, sho por cuento sropia no incuritia en bogravante. Sise tha tama do un simpie anlado. ka pena seró de peritenciario de 19 a 20 afos: pero si be tratara de uncabe cilla o ditgente de la orgarizocion. i peno sero de internomionta en vefo a lo mavor iviensidiod de su cocpetación. pirosto que en clioo reside el pernocriento que dirge la organiagcion y a mayor acthidos an ia evistencia y combión de las octos temoristas.

En razón del fesutado, ternernos

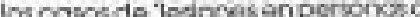
donos ro coneldarcelos on bienes

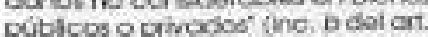
2606). de 'si el dano en los biones pubicos a privosos impikieen, fotal - paralotmenta, los servicios ogary cicies de lo peblación unc. d dol art, 20ses $\gamma$ 'cuando se caisate muerte o ledianas guver" sine fol art, 286a)

Enla agravante de lasianes en porsoncso dońncsno corskbatablos en bianes pitclicos o privodos' il by so rofiete a jas ledicries monos graves del ort, 100 det $C$. pues las bsionos graves apdrecen foinea dasicnos praves apdiecen lansa "donos no considerables" entende los doro de poca troortoncio o

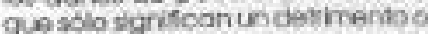
dismirucion ca la colidod o. LAF. dad del bien.

Apcrenterrente bn ley poctio llencir a la conctusión de quo el geonte debe responder cumqua

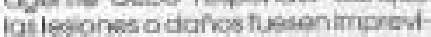
shies es decr, quese estarban osgraniso uro responsnibiddod

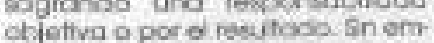
bargo de ocubida conel pirncpis cispotilitio que congos on su iriteoridind el cocigo. pecternces intogar el tento an al herilido de queé agente sdo responde cuorido las nejoncrodohos, sondo picilables. pot regigencia flatia de oterkión a do cuidado), no los haya previs. to. La pena os poritenciatia de 18 a 20 ance.

La figura agramoda de "st ol darbenics bienespuelicos oprtvo dos impidera. tokd o porcisimen lo. Ios sorvicioe esmelalss de ia pabicich:. Ie comete el que. sh cioar una shuación de peligro comin, inpictere, estarbare o m torpeciere \& nomkil funcibrs. rilento de las inrvieses piblcos de comuricocionn, de prestás de agua. do electricidad o do sustan clos energóticos $(c t, 27)$ de CP) wotendore do dornos fcustruceión

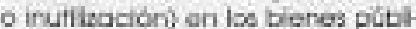
cos o privadoe. Lo quo la lay prosego scn ios semesas púliticos men cionoden, recuizados par el Estada opotconcevomerlos no ung eitun clon de pelgro, lo peno es pere - tencleoria "no menor de 20 anas. (s) cluibso que o peno manimo de peritenciaris es prectsamente $z$ arica).

La ogrovante' 'cluando 40 cost scre muerte o leviones giavce., Do fefleve cl clatito de tentorymo seculdo de muerte o lesiones graves Estos resstoctos cuatícodos cor protianifencioncies. Dowaus s tio fun abremoclos por el dale dol agonte. El delito selia de homicicle - de lesión dolossos lo perio es de internktriervio.

En azon del moclo to flase basica de lerichimo se acrova 's se tviciere participar a menotes de edod en bo comishonde desto: se tratrade un dello de terrorismespor obuso en la candiclón del 'particl. porile, un mano' da edoce ta hiry piesume jurs of dejine que la purween que en el morrwerito did he cho es unitrongt ctatedod. crorace de lo capocidad nocesurio pora eorrercnaer el sentido de la con ducla del ugerve. El coso en da ing giflang rrogiato fa monid on

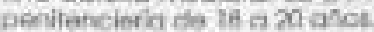

th maon del measo y th dol

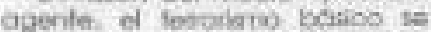
agava "cuardo con thes tartors tos ie extonioncre o secuestare pettchat pora bblenct exccreelo. crares de cetenidos o cudqule otroventcio increblsa por porte de 16 cutorisks o particulaies r cuando. corridtontica findidodi ses apsderrase fochamente de vehiculo asreo, ocustico o ternastre noclo. nd o extrankero, o alterare za itine: moria: $Q$ cuando ba extorision a se. cuatro tuMere corrn inolicod io oblención de dinero. bienes ó cualquier otra ventais".

Esto creuratoneio ogrovente spone medios deterringdos y abjelivos deteminados. $120 d$ os caubinente: Los medios san id extoricon o ol secunstro, esto os, o poder pooctwo do la prtwocibri de bertad on of rrisma. y por eto se trata de un verdadero caso do inth. ridocion o exoccion. Los objett wo scri: a) oblener encurculcicio nei de deteridoi por tamorismo b obleriar caxigyier vantola incebi. do por porte de la outcridod o particularesi e) apoderarea de vericilo careo, cacidico o tories. tro. d) atforat of ilfinororio de escot voticutos $y$ es cotervibri de the

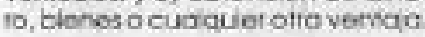

It sentido de ic apravarite estó

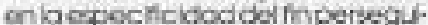
do No es nocasanio is etectivg obtencich del fr perseguido. Lo lekserefiero al simplo plopbelto de ohtener cuadquier ventag ndebt. do caso motho do la extonidn o sectiratio, con to cua pone de manifiesto quo el fir pisode ser one fograda Lo pena es de iverno miento.

Es fadoe los casos de Aerovinic agravado, adernas do to fence

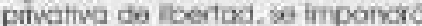

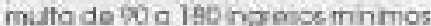
vitakes e trhotintocion absolita o interdocion civi divantela conde nay sande posiodoros a dia rait 2885)

A: CONCIERIO PARA OELINAUR

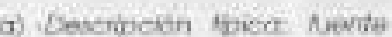
legor

E cal. 2eac defme la fiono os conciarto pova delnque en ia tor. 


\section{ma sigulente}

"Los que fomaron porte de una organizocib́n integrodo por dos o mas personcs, que se cogupen o mocien pora instigar. plonificcr. propicior, oegorizur, ditundr o $\mathrm{CO}$ meter ockos de letiorismos macho. tot oinmedictos, prowstoseniasarticulos de osto Titulo, seren reptirtdos, por el sole hecho de ogupas. 9 o crociose, como tambihn por ser miernbroe de la orgarizaciko. con pend de peritenciatis de no menor de diez arkas ni monyor de qunce:

Lo fuente legidativo de esta regia se hola en el art, st cel Decte to ifgidativo $N^{n}$ CAs y anel art, $4^{t}$ do Propecto propatado por la V Con. ferencia de la Asociación hternoeional de Berecho pend. de Madid, de 19z3.

b) Taicidod abjetha.

Cbjoto especifico do to tutab ponol, es ol interbe dol Estade ose mpedir que se foimen socisdoces cirrinosns, anccrrinodas o come ter deltee de tonarksno. con peitgroperrnonerite de la tronquedad publed

seseto activo puode ser ínicomenile ung piuridod dia personcts (per esto nos halames anto in delilo colectho), que bleley firaenel nimnero minirno de dos, a diteren-

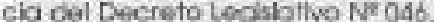
que expio el concirso de res perwonos por lo mences Se reflere quo lodas los porsoncs sean imputo. blesi por bo torta, no intercran ese nimero ios onfemon mantoles, los mencres de 18 chot, elc. Plito no se recujene que las penonos estern moteriamentes reunidas, ques in conoiccon reciprocamesta que etren unidas poe un arto fornd obc. pues trosta ef wherfo $q$ is los une de un modo duxadero $\operatorname{con} \theta$ carocter do pormianencía ques es lo que dhengua al conevecto para deinquir del imple acuerdo crimi noxs.

Scieta pospo estio sociodiod y e Entado keksnodoe en a i bien lurs.

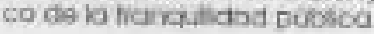

B comportariento contiste on formar parte do una organiasción ntegrodis por das o mos personcs con el in de cometer actoe de tertorismo. Por lo lanto. ei comportarnierito conata do dos olementfos: A) Formar perte de unnorgarisocián do dos o más parsonos. lo ques signifoa porticipar de una orparizoción. agarización que expresa coondinación y skemati. zocion de las cethidades do los miembros o socics durartin is consiturieion o despuest do ola. asegurando su yido y su tuncionofriento que no necresito ser perfocto. de tipo wodel, puos basta uno organibaclón rudimentaría o miritna, con fal que sed eficlente para sur cbloso de instigar, piniti. car, propticica, orgarizor, dfundro cometer actlvidodes termatstos y B) El fn de cometer octos ce tencItsmo previstos en los crilicuros de aste Titulo, na importando que so comoton o no se cometan efoctr. vancrto, pues la lay solo exige que se havg querido cemalentosi y i realmonte son cornetictes, bs asociados cupcobles sesponderon do esos como terronimo egrevode (art. 288B).

\section{C) Tpicidod rubjefiva}

El delto requirese dob. 5 seg, acusido de volistiacise de por lo menos dos sujetos conskiteriso on obsoryar cintto canducta y on conddarcras reclprocarnante unt dos. de modo dunadoro poro sols. tocere el propobito comin a tads. de cometeriunosede ndeteimno. da de actos terioratios. B oruerdo det coencista pera delnquit se de trmous def de la paticipoción ent: minosa coman.

Pera, como yo we djo, no so reatiore al conocimiento en concreto de icas pensenot. que puodien oestratrecer xecreflas con tol gio

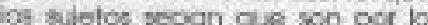
menotdoty quo doben roalarr. en el preocento o en el Autias. ciartos octlvidades fersortins:

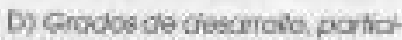

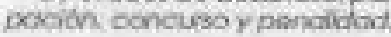

Este delto to consunis en el momerto mken en que dosomks pensonar se arrupon o crocian pora instigar, plarifica, picoliciar. organizar, áundiro cometer octos do temoriemo, es dech, cpencos so prornueve, se constihiye o se orgo-

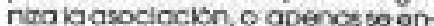
tra a tormar parte de eta. Corrose trata de un delto permanente. $k$ consumocion se probonga hasto quo la asociacibn sa acobe spot disolucison de lo asociocikn o po: detencibn de las osociodios.

to tentotho no as poethie.

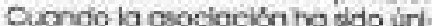
camente premovida. pero no coristituida. no hay lentattva pies in lay no na desplazedo harita cos oceón laprotoccóndel bienjurid. co corresponciente. La key no ho condiderado al coto de 'premovor", dino en vata de la csociaclón ya constituda. para espechoor una de los formas do octuidad defichuosa de los asociados.

Por ser el conciorto para delngur undelito autónomo, en odmin. ble, respecto a el, la copornicipa. cidh be persenos extitarias, sogin el art. $100 y$ s squientes del CP.

En caso de concurso do delitos. debe obvervarse lo cispuesto cn al Titulo XII del Libro Primero dal CP (art. 3" de la Ley N" 24651).

La pena es poritenclería do 10 a 15 álos y muta de 80 a 180 incresos minemos vitales e inhabil. fación abeokuta a interdiceiśn civ? durcontela condenoy 5 ches poste. nores a alla (crt. 289F).

\section{SINCEACION $Y$}

\section{APOLOGLA DEL TERROPISMO} oai

A) Descripeion filalca/ nuenta is

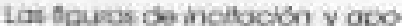
iogin dol temonwmo se hoton cer: cebidiss en el at 263D, de la s. guterte increro.

Taque putbloarnerite o a traves de cuclquer maclo de comnnico cib́ris scicil, Writare a cometer lot Cellide cesciltos on estra spccion. at como ks qua hicleren póbbco- 
mente la apologla. excitacion o elogio de un aeto de teriorimo ya comelibo o la cicbanea ce persa na o de pensonos condanorcas o sentercio fmre pot su perticipocionen hecho crirind serónneprmidos con perilencicrín no menor de crico atos:

La tuente legalativa de exto regla se halla en los crts. of y $p^{2}$ de Decreto Legislotho $N$ asb; y en of ort. 32 del prevecto de regulaciśn del temalsmo. preparado por la Asociación intemociona do Derecho penal, en su reunán de Modivid de 1995:

\section{B) rosididad abjotivo.}

Obuato escecilico de la nutelo penai, osel interwado Estadopere montenimiento de lo tronquidar DCblica contra los octoe quo fiernscrin o monoscasoriks, a causa de laincitocion of blied al tenotamoc de la cosologia del temorismo, que s.scitan sentirientos do proscupocion en od pueblo.

sutoto octiro puede ser "cusal quera

Suloto pasino os la sociedad ee Futodo, teriendo an velo ef objels juikico olenoldo.

La conclueta coniste, en la $\mathrm{h}$ pótesis de la helfocibrin on actice o en el empleo-de modica idánaot para helin a pessoncs dol píbilco (aunges seo a una wila. con tol que el hechose hoga publicurien te) a cometer bes deitios de iemorti mo previstos en la Sección Cotava TA' dol Ce.

Nickes ded fipo on neitor eito os intipor provector, encetitor. estit

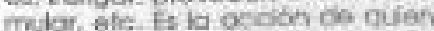
arimg oirmpeie al deito de tertoris. mo, on asto caso

No es eterrierto del tipo ín leal. moíón del delto de leviorima La iex reporne ta trictocion en if. se comesto a no of detito hallgodo.

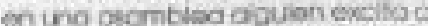
iot cyentas a inchar a ofta y ia intigocion no os obedeciga. recho ohoce il tipieidad nelcest: tio pamo la incrikrincoión. No se extge que lee oventer aceptan of propleito provocodo.

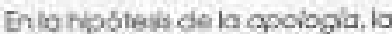
conoluetorecrisene en octes on on ampleo de mediss ibonces poxd emoltar 2ugestivomante el delito oo temeriame yo cometido o a le persena de su quitce o particioe. cor derado en sentencia frme.

Hocer lo apologia' as el núcleo del tifno, Apokecís as elogio encomia Consecuerverrente as doglor, enallecer, exditar el delito do iunodismo yo cometida o a la por. sono condenodo on sentencla tirma de modo que coreht pya und inclitacson implicito a comeler of delito de terrossmo. Es menester que el agento elogo al temorimo en $f$, o al condanceds coma tol, $\theta$, en otros polabias aploudo ei he cho protibido por la loy o a 2 gustor, No es oodocíf quiense imi. ta a lustifcar o oxplicar ib oonducio dolotuma, dotocondo emol dades o ahibisos del delncuente. en ecryapeso ai hecho crmhoso: fi karpoes laefinca o aprociocién delctipotilivo iegd o de una dect. Sen: lampoco el copyo moral ds. pencodo endetomindda: cbeurs. fancias, coms basede ocumit en relación a un 100 condenado cor prupbas ircomplatos ave dan márgon a la duda Estas actituses puedon traduer critcoto centara a la Justicla. pero no dogia del

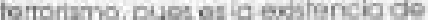
beto qua, procisamontle, en este cosos, s2 pano en dudo.

Ia opobola dorma do irefigg. elán hdiectiolid fore de la imita. cion. poraus esta porna su diencán en un aconilecimiento futura.

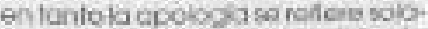

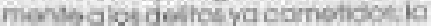
neiloctar so loga of tenorimio a comeler 10 opologiose ibociond con al terrowise ya corrvasdo y solo indeers nmenta se roduce a la ncilac on a compter fertorim?

Wh exile hatiacion al fexporme

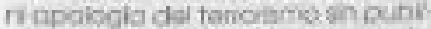
ciand le niviciciad of eterranto

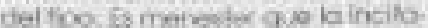
corrolacpolscistsed hectuarile cletto nimers de personcs recust. to indispersoble para que te pusdio hobiar se perturbocicen de la fonguidad puticlea. de olamna sodel Desde lyego, no es solamante al numero de personas to que calacteriza la puticidad: la incitación a terrotimno o la copologa do fertoliero hecho por a. guien er ung teunién forriar. dondo hay vorica persoras, no atrece in tibicidad rocuento. Porque be punicidad es corntituida tambisn por el lugar. d momento y otras circunatancias que toman posible la percepción por un nomens indaterminado do persohas. la incitación o la cppolggio det torrorismo

Porb tanto bincitacióncl tortotemo ola apologiadel terroriemos

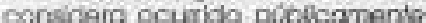
cuando se comete of por medio de la prones o por otro medio do comurkcockbri social: b) en lugar piblico o chiorto al público y en proserciode werics pentomexc) on urid reurlba que, por al itugar an que se edactici, e por el húmero de ios que an ella intorvionen, o poe ol fin u obieto de ela. Tena canacter de recrion no privoda in oud. giver ctro coso, nose podría consh derar en elhecho. lesion ol interes queccorstifuye elcobieto de is tule la penal

La puthleidad. magida vor el tpo pusdo sor procucida pot wo. fios modise in paiabra el excrito. of posto son modios id broos paro incitar al dolito de berevismo. cano dice solir. pueden serio. horta los actics slenciosornerse coptutados pero cuno santito re.

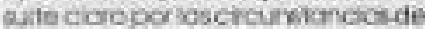
inger y torteo' (a)

\section{c) Jercibod sctowend}

A. amesto sitideitio supoos dola Hidolo cordate onia voluntoci de notora cometoractos de teno. ilimo a de hater la apologio de entos contitos, teniends cencest miento de la pubicidodde trecho.

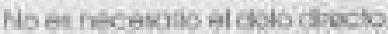
bogin eil daie evenhy prectsa mente pampue se fraila de neita. 
cisn clecta o indrecto o la comt sibn co cetos tencritos

D) Grodos de descrialo pontepoción. concurso y penalidod.

Extos delitos se corsuman no blenla inctocikno lo apolocioson pencibidos per ics pertonos o kmm bién por lo penong a kas cuaks están driaidos. Es det fodo indileronto, paralos fines de laconsumaelón. que la incitación o la cpola. go sog oceplodo a no. y on a primar exento que sor o mo segu. da del dolto incitodo

In fontafiva os admistik slam precue anvoritcarse el hecho de io publicidod, hovo octos ejecuttvor. Asit en el caso de lo netración - capolosjacoritredio de laimprenta, sil los imotocos ya Jatos odra la divigacion son socuestrodos por ias autaridador.

Sila incitosib́n o la codbgli es.

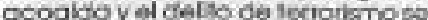
comele, se tiene coporncipactón on el delto incitado.

E concurso de dellta debo ro sobvone conforme a $k$ cispiosto on al Thulo Xis del ittro Pemato do: CP (art. $3^{\circ}$ de to Ley N 2465)). For elemplo si of delis heitado as complido como efecto que lang su causo on la vietocib́n. el tujoto iesponde en corcusso de dolías tanto por la nelación como por a delita nisiado. No os estes el parocer de RCAROO C. NUNEZ, para quen si io heitocion al ierodsrto no dopende de la tentativa o consumacion del dotto incitado. co corrision del delto de famoristno abiato derela, no daria luocr a que $\alpha$ heliodor sea un porticipe de dalto cometito (S).

Lo mimo debe decines st of detho es cometido como efiecto que Nervo su caviso en lo cpdogia.

Le pena. en orrbas hipoless. es perverncionia de 5 a 20 anca: y multo de 90 o 180 horesos minmos vilales e hinabiliasón cosechula e interdición comid diranto la conde. na $y^{5}$ ches pasterioras a elles fart. 282 ?

\section{0: AUXIUO A LAS ACTIVOADES \\ TERRORISTAS}

A) Doscripción flplear ruente iepat:

La figura de cuivilo a los octih. dodes fencristos se halla cancoht da an ol att. 2aBE del CP, de la siguionto monota:

sand raprimido con peritencicno no manor de aunce ollos in maycr do voente, ei quede monero

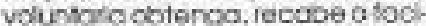
Ite euclatior acto do colabaroción que tavoreacc to comisisn de delifos comsrendidos enesto titusa - la rodibodín de los knes do un grupo terrotista.

- Senactos de colaboradón fos siquionters.

a) infomación soble porsenasy pathmarios. instalociones ad fícion putecos y privados, centros urtoonos $y$ cublesquera otros que seon tionilcativcs para lat ectividados del grupo tamorbia:

b) Corstuecion o utizacion de cupiquer tipo de cicjamento y atro elemento surceptitio de seir destinodo g ocutitocibri do perse

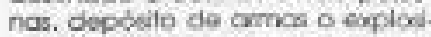
vos, vivoros, dinets u otras pertenoncica relocioncidas con fos crupos terroriatos o oon tus victimas:

C) Qcullasibn of hosiado ce personas hitegrodos en las gupces a venculodos con tha ockivisjoster delictivas y la preatocion de cuct quer lipo de onuda que tovoreicos lo thop do aquitias

क) Oiganzacion de cursos o comoos do ontronamiento do chis pos temeristos

e) foovicar. butultr, sustroer. cimacencer o turitristicar amos. muricton metonctis it dejeto tox ploctvo, intiorictile. colfsionte o tbobco: o cuciquier otho forna ocondimico o de apuda o de mediocitr hechécen io fnoriogas oe fnanciar grupat a actividodo: testoridus dey tor zabias?
"Cucndo las hechos telaciono. dos en los pourctos anteriores sean suceptibies de incriminoción con creedo o otro u otros preceptos, se cplicand el que sencle pena de mover gravedad?

La tuente logíativa de esto recla se hallo en el crt. 3 r cel 'Esto futo poro $\mathrm{k}$ defenso de la dertacrocia' de Colombio. de 1958.

\section{B) Tricidod ablotiva}

Objoto especillico do ia Aulala panal, eselinter bes dor Eatodo por la deferisa dela trongul dad publico. coctia las tochos que bo ofencien modiante aristencla o ayudo a tas parsonas que cometen actos de terpotisno.

sulato activo puade ser cual. avier persong que obtenga. recabe o tasilse cuelauer colobsersción qus foverescco la comelion de actos temoristos.

S.yeto parvo es is colectividod.

E) comportamionto impica cblener lecostor o faciltar cuat. cuior acto de coloboracion que sovorosco la comisón de deltas comprondidos en el istils o la reoIaxción do los fínes de un grupo terrarista:

Núcleo del tipo ea obtaner, nocabar o focittar. Cotener es od quitr, lograr. contegur un ocio do codabaraclón letrorista. Aecaba es recoundor, solicitar, endgr, ponel bir, osegunar cusotos pecuriatias o en urpecis o de cuciquice otro indole para fermoremo facilitor es potzillar o smpificar b ejecucion de cigo o el logro do un finc propor cioncirmedics o recurscs con tree: ternolistos en simp sa trota de

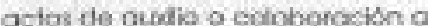
ias actividodes tetroristos

to ley condidera cerrooctos de colaboración a las octvidodien lentonatos, Vsesiguenvas a) sumnis frut informoción a fombitilas sobie peisones y pahimorio, limlónci.

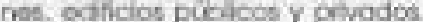
obtvion uibarios y ahos sigrificos: 
vos pora las aclividades del gupo tencrista: b) La constucciarn, ceson, utlitacosión o arrandamianto de cudauor fipo de aclarniento. inrrueble o edificio susceptibie de ser destrocido a ocultar pericnas. depsuto che armas o explestras. viveres, divero uoticspertenenclas de kes crupos terteristos: c) Doultar - Nasiodor personas ivegrantes. de grupos terroristos a ynctiodas con sus actividodes delictivas y io prestación do cuclnuer fros de onuda que favarezca ia $n y p a$ de coqublas; of instrucción y entenomianto farronistos meciante olporiboción de cursos o compos de entrengriento de gupes tencris. tas; $y$ e) forotecimionio temorictes. fabrica, codedir, sustroet, almoce. rot o sumintstick ontics. municiton sustarcio u obieto explatho intomoble, cstiviarite a fíxico: o finan ciomiento femonisfa, cualquiler otra fornoeconómka odoaycia ode meclocken heche con io fraldod do frransiou en pros o actividades tomcristos:

\section{C) Tpicisod ubatentar}

La concuota det ogento debe sor dolosa. It dolo corresto on ia volunfod de colabercr en ia corre eton de cel los de terrovismo o an ic reclipociónde kos fines de un grupo scoraristo. a scliendas que la persona hotheduat o en grupo oudikdeporticiog enesas cetiviobodos. y conel finde pisestarie crudo o ors tencia.

La coocción o el enoc oconce

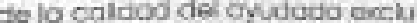
van bo puntbilided del feche.

D) Gerodes des deoxmala porich nocion, concuso y nemolood

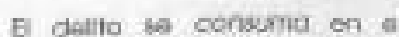
momento y hogr err que oblergo tecorbe o tacilie cualauior octo ds coloborocion oue favokesca la pomishon de delsot de terroriand Esmenesterauelacoloborocibris toog efoctive Como esto deito no se tanesinose da owhencide

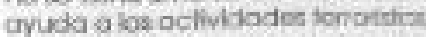
pot exlonose corcums siciasic do nose therafisto de eso
Io toritativa er posiole, ciando se hava desplegodo una conduc 1apecutwa, pecoestresuladonase ha vorlscado: por ojamplo, el cak colsueto quo di levarla intoma cibn cicstemoristoses ceteridopci is policio.

R ade haber participación de un sercero que diectamente ro presto la colctogiación. pero que de und 4 otsa manero contrbuke a quese oto la realice.

B concurso de dollos debe vo sclverse confotrine a lo clesuesto en ol titus Xill dod Libro Prrmero do CP (ert It do ko Lay $\mathrm{N}^{2} 24551$ ): y al tespecto b uitrno parte cel at. $2 \mathrm{eb}$ estcblecro que en tocos aquolias hipctess en las que lss actoe de coloboración o kes activtdodes terroristos 'seoneriscepticles de incrimmación cen canoglo dotto u otros preceptos se aplicand of que senon ia peina cse mavor gra. vectod"

Lapenaespenitenciotado 15 a 20 chos: minta de 90 a 150 inprosoc midimes vialos e inhabilucion coscluta e interckecion chal durunte $x j$ condena $\gamma 6$ anos postricren a ellosort zasf).

\section{1) EXMENIES O ATENUANTES}

Eximentes o olerxuantes de res

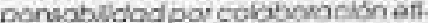
caz.

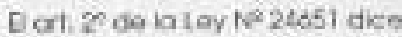
be siguento:

'Atickssose ol squante articuo a Tulb $x$ del Ubro firmero der Codigo Pencl:

tArt Bas. fnilos conilos cte ierro. sumo sonan chcur tandas entrem? tos a ctancuantes pora la procejo dion inctivazal de xu perval ion ilgientes:

0) Que el sueto rova coanoonorkx vilutyioments az activi. dodos doliof mosy he prosonitn atio

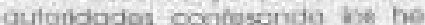
chos an 9 s nutbere parl kcipodo.

D) Suset ahandono par olcu- pabla da sy yncuación crimina hublere exthods o cisrínuldo va. tonclaimerte ung situosion do peligro o mpencido la pred. xeción del resithodo cloncsos o cecinivo. do eficcumante o lo cbtencibri de pruebas decivivos paro is idersis. coribri e contura de otros respon. scibles:

(e) En las suowestos menciono. dos en las opcrtodos onseriotes $\theta$ frbunalimpondru penoinferiox o is fladd pard el delite Askisme. podta coovdar la semision totol de la pena cuondo la colobonocich actha del roo hutiero tenicds una particusar trascenderkio parc io idontificación de los delincisontos. paro evilur el deallo a poro imperdr in achuadion o el descriolo de kes grupes terroristas, sompre que for selghayosancionocso ct mis no.ec? concepto de ortes. por acciccies quo hutievers producioblo muerte de agung persona o kesbries gra ves. En este ufima cose ia pena

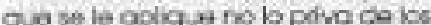
beneficios de la bortad peoviab. nol, semuleerted, bertad vigiada ceduccion do ia porno porel froboio o el estudio, corrmutactón de ía pena o indito. Esta zarrisbon quedand condkicnoda aque el tea ma vuelva a corrneler cuaktake de los caltos previstos en esta Ley: $y$.

d) B integrante. coloborodse o cooperiador do cripos ferreristex quess enciuentre en oribonconcta ngdo por sertonc io fitrue. poora cteteren is sbeckod cencicienal st

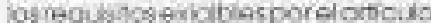
55 del Cadjo Penal. 1 concune dgurvadelos ciraustonciosa que in refore el coatods by de este articuas".

La fionto lapolide estaropja en derta foma, so hala en ol art, 3 ? del 'Estatuto para los doterisa de la demenociat de Columbia de 1908

Fe nato de moclorisodes or arutanclales que anteceden acarcrarkan o supdaueriala condirtb spisay aim tionen la vitud de inuir sorarablamente en a sagiretrig ounso 


\section{Les hipotests son kas siguentes:}

A) Presentockbri voluntono o be arlenidodet curresondasts octow. dodes terronstas (art.85A, ne: a delCP) La interupeićn olasspent sion del processo ejecultivo Mentatva inocobodoly ola no verineoción delresul nado (tentativa cecabada). puede sucedor por dos ćdenos de cousas: a) causas dependiontes de la voluntod del ajonte; b) cou. ass cienas a su voluntod ferturicas, predispuostos u opuestos POA otros). En is primara hpoteds pue do darso: a) quo la voluntod des aponte obro antes que se horio cumpldo lo occión (tentativa ho. caboda). y ontorcos fonomes el dosistimionto voluntana b) que ia vohritod, ya cumplda la acción. loge solo impecit a resutado (Dentatva ocobado), Y entorncea se liene el Iomodo amesentrrion? to gctho o cartapentmiento afcaz (ant 96 del CP) Can tocios los lege laciones del mundo peconocon vabor di detistimiento yd orrepent|miknto, pues lo hacon sogut, o de ung impls disminución de peno o de bimcuridad totd del ogente Eito. porquo dosde el punto de visto de io poitica criminal, de un bolo. se chearvo quis of que se dedste o arepciente de unabceion dolichuosa demustia menos mat dod y ciertamente memorpetioros: dod que ol que periste en ellary de otra parts, es interes del Estodio extimuior estos 'recessos" del proce se penal puomotiondo lo mpuni dad, o un trato mos berikno, a que te detene anto las uma consacuennizat de la mata obra De chila frase de HRMAZ VONUICT 'A deincuente qua se desiste o arrepionte. puente de cro't

El ogente cue intenta o coneu ma un desto de terrorismo y luego ifjesde his yevadi icaccion dola vistricio se pessento arite los cuton dodes y condose leshectiot en ace hutiere posticipods, dernusethd areconthinisto o dessirniento de ejdellto a porlomerxos una perso notbod ostuctuado poms asind kiplopia insponoctididod En este.

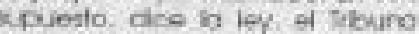
moxndid al pena niteticra a fifp. do poro of deits, oieo perna bot dibajo del minmo legal: o b) el perocon de ispena encass da que a coloberescion oclvo dot res haya servico paja la idertificcolón de los deincuertes. pora evilar es dello o pora impedr la actucisín o dexartolo de los gupos fercis. tos.semprey youando nose le hava tancionodo a opente. 'en con. cepto de outce. por dociones quo hibieten procucido la muerte do digung persong o losicnes graves? Poro on este CAtino cono, cucnde so hubloion producido la muserta de olguna persona o kejonos gra ves', la pona que so le aplque no lopriva do los benefcras de ko boet. fad proviciond. semiloertad. kborlad valodo, reduceión de la perna por el trobcio o el estudio, conmutación de la pong o hdisto' remson que quediord condicionadan que el reo no welvo o compter cuctiquiera de las deitos previstos en la Secobon Ootavio' 'A. dal on (art. 85A, inc. cder CP)

B) Abondorno de la whecubeion terrarieta que evita o disminisoung

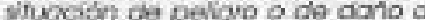
coodruna g bo obtencib́n do prue. bas. cort 85A, he, b del CPs. Se encuentra en esto shyacion de te

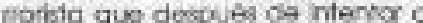
coramer el dotito voluteriamen. to es dect. por mativos exclueno. mante uryon y no sigoridos por ctto se operta do su vinclíscibr terroristo yole fstaminnara: a) evita o disminupe una situoción de petgro b) impicle bo produccion de wr resurpolo dionoso o c) cogatrervad la chtención de priebor para idertificacion e contua do olmi responsobies. cosceste utrino qus no es propicmente un crecentmiento active o eferot, sino presicmento in amepentivivanto pas fichum: quo no intervione on of curso del delta dro qua sobcente. he o wu ecrisumocion, y lo ya he char ro puedis deshoconsa.

En esto tupuesto la akención o oterugeion de pona quoda tuetu

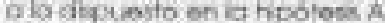

C) Leerfact conational de ked ferchitos uxeussos an ef vie $b$ die art. A.SA so trotad del aitoro patici peconviervodo pcreantancla thrne de serrorsmo que al hacer aban. dono de sir virculadión terorsta. avito o deminuve una stuación de potgro o impida la proctucción de un dinno o coadtuve a la obton. cich de pruebcas pora idontecaro caphurar o otres nespornative En este supuesto, podric obtener sulf. bertod condidiond sh los roquistos de tempo y de condacto eriolves pot el art. 54 dal CP. concorconle con al at 55 del CEP

\section{2') ASPECTO PROCESAt} as.

A) Normas procesoles poikia-

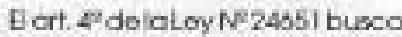
profecoer a la porsorra dal temorista frento ol postie chuzo cal poder o do la autodidod. Dice cerl:

Para la imastogción de los doltos tipificodes en esto feccich. las fusorzos Policides odoptarcin las sjousntes macidas sin peryicio do la iniciotiva que les cerresponde do ocuordo con sua reepectivgs: Leyes Organicas:

a) Efectuar la datención preventivo de lospresuntosimclicodos cemo outorest o parícices, por un lérmino no mavor do a hinco das naturalos, con cargo de dar rme. diasa cuenta por eecrifo al Mriste. so Publico y of Jisaz instuctor, arvier Se vercoerse las veinticuatio horos contados dosto la de tencikn. o en al tormino do la clistancio.

b) Disponer of irmeciato reco. nocmiento mbdico lagal del dete. nido, en el fúmrino de ila distancio. in poriucio del teconocmierito por un modico porticunor cue pur dere solicilar ef propio datenido. vi Abogado o cualauiora de kus familionex:

C) Trosladar et detenisio de ur ugry o otio de la Roputolica doe pisti de efoctyados los reconod. riervas mbecicos a les quo no ro ho

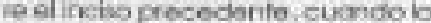
mendido pois eificlorrents nece. solorrya eimejor bxto delairyes. 160 isn polesd a la seguridad del deterido. In cutondod que dispo ne of troskado informand perevia 
Inenfe y por escrito a juez carpo. fente. oxpresando ios rozines qui istifiquen to oxoncion ola asto medida. El trcalodono podia exce cior dal plazo schalesdo en el ncixo b) de este articuso. Asimsmo, is cutoridad pandh of traskato of conocirricrito del Mristerio Puth co del hagar de destro:

Esto regla reproduce, ocal tex. Nolmente ef art, 9 sel Desietc Logustativo $\mathrm{N}^{2}$ 04c.

L thascondercia cue ferper la vido social la inoulación de ia comidion del delito de ternorlomo. sobie tocio cuando se is restingeie tbertad a una persoria cerno con

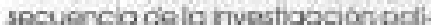
cial, moliva cue tanto la Constih. clón como las leves procesake oforguen al impulacto centro ch procecimento poled. una serie de gatanflos quo debencin serie netpehodas en b abecluto parcina dor causo a gin fes merncs secon vidodos y dé boseg a boceien do haneas corpus

Ente in gracrisas a las que

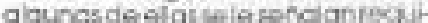
stos para que puedan eurgir tin efactos juriokos, el ort. $e^{2}$ coreida ra como tunciomentcies las sguientes

O) Lepoicio no pude derenar a temerstopormde do is dias. dandcuensa ch elo al Mristetis fublod y cal Jueg vintseter antes de tas 24 hous de proctixito ba ditencion.

b) La poficio. dentio oel termino de lo cistoncla debe somerar al defericle of un examion por ice medices boglstas acerpa de sil 10 lid fiteo r montal, an pericicio cie toconseimionso par un médico

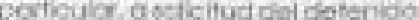
su Abogodo a cuaglater familiar.

Chte puificia puorbs trasicada of

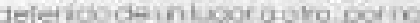

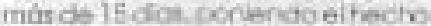

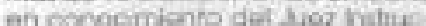
for y edel Miristodio poeitid con excresith de kat razonos para ese

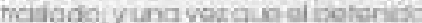
Geove el kigar dirde tha kapolein ponciót el fectio en conocímento des Mribotorio Purlico.

Fstal goranfos solamente los puoda gor un Exocos, cuancle

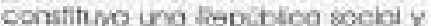
demaciálica de Derectio lat 79 dela Conthuión.

\section{b) Nomas de procedartiento}

LaLerN 24700 , de Pde juriose 1987 estatiloces un tramite ecpeca pooca lo imesigosón y jungorrien

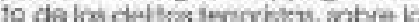
bese del jukio pend ordinotio Por condgiente, en este piosedi. miento, a folfo o sioncio de la lor. especto dis un trarrite doxo, 90 covecon las recolas del fiscio peno? ercinorio, que son siplotorios iarts $3 \times 6)$

Fi procedirneltho, en sys diver sos periocios. 9 A sencio in les 24700, fo la tiguente:

1. Prows o preshninur, earoston xada por dos claser de activalo dest cotiviolod investigndom?

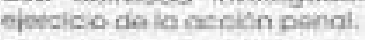

La actividgo imestigadocat ath

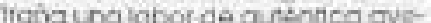

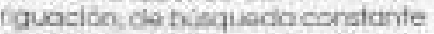
do kas pruabes que cocreditan $\mathrm{ka}$ axktencio do bs destos y la foe pormestiting do giveruet in elcs porticipan. Dxeante eska octividod re ocservara bagianorite:

- Forma e f Fecal Provincial se encorgand dirotamento den hir hestigacion on delense de la topo ctari ge los derecties twingrins dejosintateses hitelorsesper lo ley Whe inainter os des las Fueracas Fol ciutes particionon y action eri kt

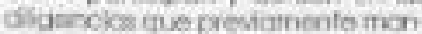
da el flesel (at, 2 sogundo parso)

- Comericackandifacol de for no. $A$ serideletida a oervinckida

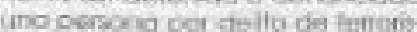

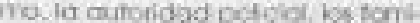
iorosdel dehantionoksocmilene. de Escochai Himconce correri fanin de irerootalo y por escitt:

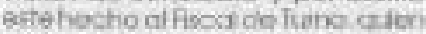

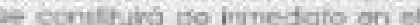
Hagripue tend un centio ofect de defencioni in peticia coniuricaí de eate hecho oxi escrib a lo pervara aue of deteride indiaue iart 2 . phimera porte?

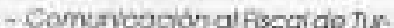
no. Al ser detenida o dorunciach una pesturva por delito do terrarí. mo, la autoriksod poleial. loe farn igres delcletersico o kas comiciones de parechas Humarice ecrmun: conori de himedolo y por escrito este hecto ol Fisca co furno auson se constituná do irtmodiato on of luyper cuse send yn contro oficialde detencion La policía comunicave de este toctio por escrito a la per: isena quo ol dateritio hidquo (ort, 2. primera pate).

- Participacien colgotoria dal Abogosto delerea. Es indsporas. tio la parlickoción dal Atbogeso deforeoc on todas y cada ume de ka difgenclas que se practiquen. El derecho oladeferea es menuriaDie. 5 por ciuslauer creurstaricio al Alyosoxto deternor faltaro a do:

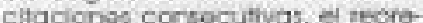
sontanto del Mristeris PAtiso

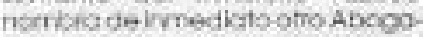
cs deternor \{art.2, temenra parta)

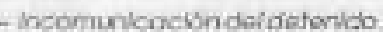

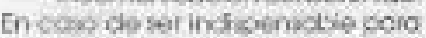
elencligngcimenso del delto, of Fo

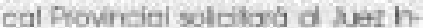

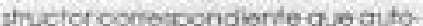
tico id nocmurycasiba ded deter? do par un picaso no mavar de ciè. clat te nccmurvcocibn roimpide of canfercucias on piringdo ente il Abocosdo dotoriast y of delentdo. los cudou no podtanter proth bistas iper la Autoticiad Paficici on

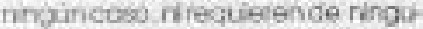

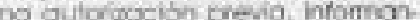
do alfiecai Powineis.

iosogindancliviobofouretro-

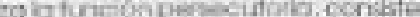

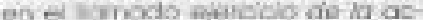

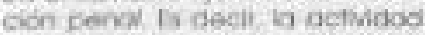
raveltigadan pejede ongirar ias ixguentes consece ancicis infolcas

Conching iq invostigacidin

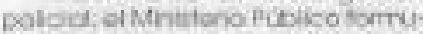

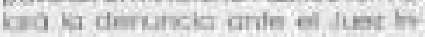

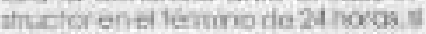

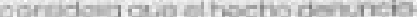

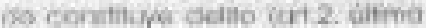
perta) 
-3 de los elementos aportados a la imvestigocion previo o prairr. nar, nopuode ejencilarse ka acción pend por que eif fecto que motiva a clankincio mosenconitihtho de doito se cesendatá el crchivo do $k$ ach xada.

3. Vustrucción, quo conckite en Gveriguar la existencio de las det. tos. sos circurstancios en cue thr. bieren sido corratidos y lo respon. sabidad o lrempersictalidad do loe incupoder se cbenvorín las at grieriles roglar:

- Jueces rutructones a dedica ciôn ercelusiva. La instruxeión pusde estar a cargo do duecesirotructores a dedicacion exclusiva, dosignodes por las Corlos Siperices ropoctevas, die ocuerdo of fos dis. pceiclonxas cue pand id afecto estobleico la Certe Suprema de Juficia der la Pepúbica, en los casos Qua tuero necescrio (art.3' primect pate).

- Auto apertiorie de refucción Recibida is denuncia par el Jue?

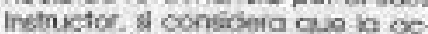
cicin penal ne ha presct to, qua el hocho derzinciado constituve detho $y$ que so ha induktuclpocto at outor a autores, dicso ol auto opertorio de iratrucedon con orden dp dotención, en el Nemino do 24 horas (art $3^{2}$; ine a).

- Auto do NO ma lusar. 5 el Jupz instructor corvidora que no procede 10 oscion parycl, ol auto corresponcliente seno elevodo en corveuta al Tibural Oarrecsional dontro del mismo lesmino. conttnuando la cetenciben dod deniuncloss haslo que ed inibunal ctecuiol. va el crodo (Ait anc b), E tifounal Correccional cubsclveto al grado en el fómríno do tros glos pievie. disctarnen dol Facd Sipotiot, que serd evocuodo as el migro ferrt. no (art, 3 , ric.e)

- Intervericion chicutona det Fiscd y del Arograds. fi Fiscal fro vincid yefotrogodo defenscr her. venctian -obtogitcriamerite an fodus le digurnitios de $b$ instho. don.
- Incormunicación del imputado. Cuando buete indisporatable paraks fresirvestigalorios, ol Juez mantendro incomunicodo dincu. pode, eun dospube do prostedo la retructho. io que no excodond de dias clas.

La incornuricacion no impide icxconferencias entre ol incupode $y$ su deterisor.

B Juoz hetwictor da coviso de la nocruricacion al In hunal Cortreclorkal y axprescida lcs ranones que haya forvido paro ordenario (cat.4).

- Volder de los digencias pol. cioles. Losdijgencias ochuadospor la Polcia, con la intervanciben dal Mristodio pilbico y bo dotenso. no se recsetioninen birstruccion scaho. la deckacción orrolatoria des incupodo. eucindo el juez la cons: dere comveriente con, 4. Baptrixa portes)

- Duracion de la hatrucción. Lo intuccionconcluivienel pictos de SD cias (at 20 , a intaparle)

- Prérioga dal plaso de inatruc. sibin. 9 plono do la hatruceicin podta ser premogedta o b umo por 30 dics mas. cuardo por el furrioro de incupadot o por no hatesrse podido octuer pruebas considarcyos sutoncicles por al Psod. per cousal no atribubir a juez a al fecal, fuera necescrio bocerlo por opiriaras contomas de uno y de otro, creproscascs indepencierverronle (ast. $4^{\circ}$, seasa porte).

A. Vtermedis o de transito, que toro por finclidixy el aute lospartes precken su pceicon, basandose

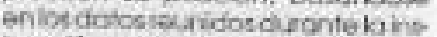
fuccion, en cecr que ol Mricterio Putuco preces wi mecitades y el

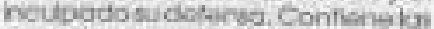
icrulertes moditicacioner

Conbcirriento ge ks interisa das. Yereiss at Plazo erdingila. en su caso. Et pitritogadio de lo histucebon. se porniro a diepodi.

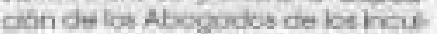

pados y de la perte civé, sirnusto. nagmente. por 3 dias en cl Daspacho der Juez (ort.5)

- Dictornent Fiscol o irtsome dol Juez. El Jisez hatuctor temtite io

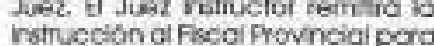
que exrilo dictamen sctore bu món 6. en el clozo de 3 diox y, a suryez el Juez inetruetor emifiḱs su respoc. tivo informe dentro do los 3 dilas vigulentes (at. $\left..5^{2}\right)$.

- Elowación al Tequnali, In irs. mucciorn serd olovada hdefoct blernerite al Titound Correccional at dècrno dia naturd do voncido

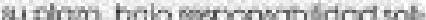
ctorio del Juaz y dol fleccil Prowincial (art.5\%)

N. Aagamkerto, que terne pa Inoldod gue tos partes so hagan oit del Thbured Conescional. ros. pocto da la stivacion que han sce. terido en los periodos anteroras dol peocecimionto. So sujetaid a las roglos dol Lbro Tercero del CPP encuanto le soo coplicable, conics siousentes mocificacionss:

- Tribunales Careccionales Ex. haofdhances. El jugarniento podrc ostar a cargo do trbeanclas Compc. clcnolas desigrodos por la Carte supvermo. tervends en cuento los requesiventos plocesalos qua so presenton a rivai nocional los que

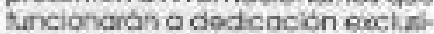
wo cribe catos que fuera nacescrio (ant.d' primera parto).

- Limitacion de in puabiciclacy E

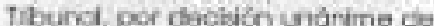
sus miembros dispondia \& ef juscio crat, ouno parto de da sesurtioncie en privado fat $\beta^{\prime}$, seacuro partek.

- Jiatgamiento en el estabiectmiento penol E Trilouna por tro yonta podro a faponer komeron que ejucio ond remolce enelestably. ciriento pernal dondess erciven fie deferiso al pnocesado fait. 6 ? segunda parto?

- Aisto de NPHA LMGARgeretciarionto $\mathrm{E}$ auto quo doclara no

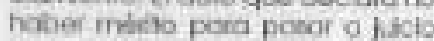
ord se elewain en constio. bais 
resoontabliclad Fte trerrita no in. pich et excricelerriento del pro ceoodo evero bertod hubienositu docrotada por el Trlound (ot. 8 )

- Principlo de conthusdod en ke audioncia. instelodo ef Tribungl funcionará on sotich ecothus (art 69

- Limite dela intempcién. Pus do ol Tribunclintemumpi la cuuction ciasdio por mosvas tundodos y por no mos do 3 cias fat $\sigma^{6}$ )

- Suatitucion csst dofensor inase. tento. La inosidencia dol cotenser do acusado a dos ouslenclos coneocutives no fusharó el zivicio eret st el procesado no designa o ofro Abogoclo dentro do loc J dica, sard reemplazado por el ctaferver do óleio (crt $\left.7^{\prime \prime}\right)$

- interrcasitas io diectoe acusa doe. testigos o perilos Puedenime nojar ciroctomente a los ociso. cod testigos. peitros, ofe. lanks e fercal como el Abogudo deferiser (artion)

- Limsacián del irtenogotorio Loe Vocales solointertogaronsobre puntos que no holyon pregsitad el Fixad o bi etro Vocal (art BD?

- Lectura de plasas. Duncrite e yicio orol, se berén solo ka piozas

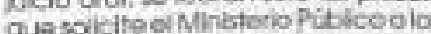
defenoa pussde nochasatos ol fis

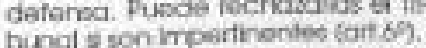

Protibléón do vecuma, No so

acining. bolo respanscbildod. recurie alguno quo entopezco en recurso aiguno que entop ezcra al (art 6r)

Y. imougocisn. qus tiene per finclidod ed que kat parfos precosen is podcion resesecto de b insciu

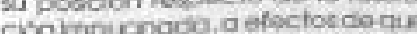
ef Inturis ciplarto lo ievise y ie

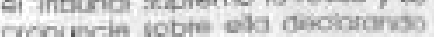

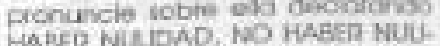
HaEER NULIPAD, ho HABER NUU-

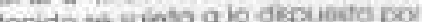
of CPp ceonios ibuientea mosifico. cicres:

- Ricums de nuendod. Controlas sentoncios preceade el recurso de rufdad fste trimite ne mpide al excorcelarvierito del procesdo que hiticre ddo abvuelso (artes"

- Vesa al Freal supremo en lo Ponat in sola Pend de la Carte suprema luogo do rocibida las outos. los posaro a vista del Fisco supremo en bo Panct, Quian emitito au cictarrien on ef plaso do 5 de: (at. 5 ).

- Tradado dilAbogado doforeor Del dictaman secol 20 corretd lio: loo a Abogado dedonsor curee doboed ahsolverio on et ploxes múxirno do 5 dios (art,9?)

- Decignación de la caza. y resolución Con el clegato det Abogocbo cetersoc osnetrarecu.

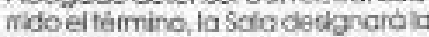
vato de la causa dentra de un clazo no muyor de 5 clas y resol. verd en el lapso oe $10 \mathrm{dras}$

v. Epcuesen procesal; quo to mifere a la elecurión cio la renten

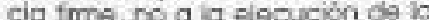
pona, cen ei fin de conzegur ef restatisecitients dedosdenuividos. pertuboso. Su conterido es of sf guento:

- Berribion de qufos. El Juez Ire. inuctar, al liburiol Combecions e fiscal Proukciol y of Ascol Su pertica. en su caso en respongat bes de ka iotrisibn ivmadiato do los cutos a lat instanclas conespondented.

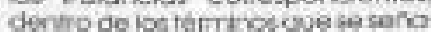
ian on la pabeurste lery fort $10 x$

C) Dpoeiclanes cartnnect

Enebte procesfimicnto obpocict.

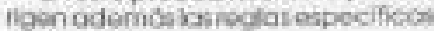
scgikantes

- Potricasien de Atestadoe lis

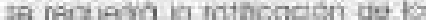

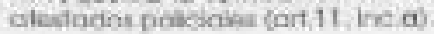

- Plecivociós do Jusces y Vo celes tos Juoces instructorist y lo Woxcins de los Tithurvies Conecianciese dodicodos a lus procasos poe lerrotismo con preterercka aneriuta o excluswomonfe scio pupden ser recunodos s del atestado polcial resulto estoclecido cue han sido agraviados por el dol to cue por haberio presenciado deben deciarar como testigos, y en tbe dornce cosos del art. 29 de CPP, requindindoese pruoba instry. mentel de la crueci clecado paro su ockridion (art.11, nc.b).

- Excorodelos Fiscales la excin an de los Fxecles solo procede en los mornos cusos de nesacibinde juscer inetructores y vocoles de irburcoles Correcelenclas (art.11 inc b).

- Improcersenda de benoficios pentonciarios. ef at St de la Loy N24051 proscrbe que los condenodos por tomodsmo no tienen derectho a kes berveficios peritendianos que son therlad condiciorat (ort. 55-59 dal CEP), som-iberlod (ant 54 csel CSp), lhertad vigia: da far 51 de CEP), rodencion do pervoporel tichojo oniestudio (at. 53 clel CEPY o conmutoción do penos, sclvo los casos de axancib́n a gienuacion de perna $y$ asorgamikento de Itsertad condicionol. crevistos en los incisca (c), $y$ d) del at BSA derCP sat 12 . Plensa ioloy cue ei cerdon puclico raferictada omnetia y al inctuto. debe montenerge carno trecio de lberoción para cuando la paz sociar seo sertobtockita $y$ ontonces deweroge Iresesano manterer iq peng in puestan la arnenaza de su aples. coin. 